UCT-TP-246/97

December 1997

\title{
The Strange-Quark Mass from QCD Sum Rules in the Pseudoscalar Channel
}

\author{
C. A. Dominguez ${ }^{(a)}$, L. Pirovano ${ }^{(a)}$, and K. Schilcher ${ }^{(b)}$ \\ ${ }^{(a)}$ Institute of Theoretical Physics and Astrophysics \\ University of Cape Town, Rondebosch 7700, South Africa \\ ${ }^{(b)}$ Institut für Physik, Johannes Gutenberg-Universität \\ Staudingerweg 7, D-55099 Mainz, Germany
}

\begin{abstract}
QCD Laplace transform sum rules, involving the axial-vector current divergences, are used in order to determine the strange quark mass. The two-point function is known in QCD up to four loops in perturbation theory, and up to dimension-six in the non-perturbative sector. The hadronic spectral function is reconstructed using threshold normalization from chiral symmetry, together with experimental data for the two radial excitations of the kaon. The result for the running strange quark mass, in the $\overline{M S}$ scheme at a scale of $1 \mathrm{GeV}^{2}$ is: $\bar{m}_{s}\left(1 \mathrm{GeV}^{2}\right)=155 \pm 25 \mathrm{MeV}$.
\end{abstract}


In spite of many attempts [1]-8] to improve the accuracy of QCD sum rule determinations of the strange quark mass, present uncertainties still remain uncomfortably large. This is a serious limiting factor affecting areas such as kaon-physics and CP violation, which depend strongly on $m_{s}$. One of the determinations believed to be among the most accurate was the one that combined the current algebra ratio 9]

$$
\frac{m_{s}}{m_{u}+m_{d}}=12.6 \pm 0.5
$$

together with a QCD sum rule determination of $\left(m_{u}+m_{d}\right)$. The latter was discussed some years ago [2] in the framework of QCD Finite Energy Sum Rules (FESR) in the pseudoscalar channel, at the two-loop level in perturbative QCD, and including non-perturbative condensates up to dimension-six, with the result

$$
\left(m_{u}+m_{d}\right)(1 \mathrm{GeV})=15.5 \pm 2.0 \mathrm{MeV} .
$$

This result, together with Eq.(1), implies

$$
m_{s}(1 \mathrm{GeV})=195 \pm 28 \mathrm{MeV} .
$$

A recent re-analysis [8] of the same QCD sum rules, but including the next (3-loop) order in perturbation theory, and a somewhat different hadronic spectral function, obtains

$$
\left(m_{u}+m_{d}\right)(1 \mathrm{GeV})=12.0 \pm 2.5 \mathrm{MeV},
$$

which, using Eq.(1), leads to

$$
m_{s}(1 \mathrm{GeV})=151 \pm 32 \mathrm{MeV} .
$$

The same raw data for resonance masses and widths, plus the same threshold normalization from chiral perturbation theory, has been used in both analyses [2] and [8]. The difference in the results cannot be accounted for by the inclusion (or not) of the 3-loop perturbative QCD contribution, which amounts to a reduction of the 2-loop result, Eq.(2), of only a few percent. Instead, this difference stems mainly from the different reconstructions of the spectral function from the resonance data; with a more elaborate functional form being 
adopted in [8]. This reveals a type of systematic uncertainty of the QCD sum rule method, that will cease to be an uncertainty only after the pseudoscalar hadronic spectral function is measured directly and accurately (e.g. from tau-lepton decays). In the absence of such direct data, it is not possible to exclude the presence of a background (constructive or destructive) beyond that implicit in the chiral normalization. Also, this overall normalization could assume a non-standard value as advocated in [10]. Hence, at present, both results for $m_{s}$, Eqs. (3) and (5), are equally acceptable, and taken together provide a measure of underlying systematic uncertainties.

A second alternative is based on QCD sum rules involving the correlator of the strange vector current divergences. In this case, the availability of experimental data on $K-\pi$ phase shifts [1] allows, in principle, for a reconstruction of the hadronic spectral function in this channel, from threshold up to $s \simeq 7 \mathrm{GeV}^{2}$. In both [四] and [5], the functional form chosen for this reconstruction consisted of a superposition of two Breit-Wigner resonances, corresponding to the $K_{0}^{*}(1430)$ and the $K_{0}^{*}(1950)$ [12], normalized at threshold according to conventional chiral-symmetry. It was argued in [4] and [5] that the non-resonant background implicit in this threshold normalization was important to achieve a good fit to the $K-\pi$ phase shifts. In [4] and [5], the relevant correlator was calculated in perturbative QCD at the 3-loop level, with mass corrections up to the quartic order, and including non-perturbative quark and gluon vacuum condensates up to dimension four (the $d=6$ condensates are numerically unimportant and can be safely neglected [5]). Using Laplace transform sum rules, the results for the strange quark mass thus obtained were

$$
m_{s}(1 \mathrm{GeV})=\begin{aligned}
& 171 \pm 15 \mathrm{MeV} \quad([4]) \\
& 178 \pm 18 \mathrm{MeV} \quad([5])
\end{aligned}
$$

The errors reflect uncertainties in the experimental data, in the value of $\Lambda_{\mathrm{QCD}}\left(\Lambda_{\mathrm{QCD}} \simeq\right.$ $200-500 \mathrm{MeV})$, in the continuum threshold $s_{0}\left(s_{0} \simeq 6-7 \mathrm{GeV}^{2}\right)$, and in the values of the vacuum condensates.

As mentioned earlier, an example of a potential systematic error affecting these results would be the presence of a background, beyond the one implicit in the chiral-symmetry 
normalization of the hadronic spectral function at threshold. Obviously, this is not included in (6). A reanalysis of this QCD sum rule determination of $m_{s}$ [7] has uncovered this uncertainty. In fact, it is claimed in [7] that by using the Omnes representation to relate the spectral function to the $K-\pi$ phase shifts, it is necessary to include a background interfering destructively with the resonances. As a result, the hadronic spectral function is considerably smaller than that used in [4]-[5]. This in turn implies smaller values of $m_{s}$, viz.

$$
m_{s}(1 \mathrm{GeV})=140 \pm 20 \mathrm{MeV}
$$

Still another source of systematic uncertainty, this time of a theoretical nature, has been unveiled in [6]. This has to do with the following two possibilities: (a) expand the QCD correlator in inverse logarithms of the momentum transfer $Q^{2}$, or (b) expand only in terms of powers of the strong coupling $\alpha_{s}$. Similarly, after Laplace transforming the correlator one faces the same problem, except that the momentum transfer is being replaced by the Laplace variable $M^{2}$. It has been argued in [6] that it makes more sense to make full use of the perturbative expansions of the quark mass and coupling (known to 4-loop order), and hence not to expand them. Numerically, it turns out that the non-expanded expression is far more stable than the truncated one, when moving from one order in perturbation theory to the next. This fact lends strong support to the non-expanded alternative. In addition, as shown in [6], logarithmic truncation can lead to sizable overestimates of radiative corrections. This in turn implies an underestimate of the quark mass. In fact, after using untruncated expressions, together with the same hadronic spectral function parametrization as in [四]-[5], the authors of [6] find

$$
m_{s}(1 \mathrm{GeV})=203 \pm 20 \mathrm{MeV}
$$

to be compared with the results (6) obtained from truncated expressions. Until the questions of truncation, and of the correct form of the hadronic spectral function become satisfactorily settled, one should take the value (8) together with (6), and include (7) as well. This would give $m_{s}(1 \mathrm{GeV})=170 \pm 50 \mathrm{MeV}$, a rather inaccurate, albeit realistic 
result.

In this note we discuss a direct determination of $m_{s}$ using QCD Laplace sum rules in the pseudoscalar channel, i.e. involving the correlator

$$
\psi_{5}\left(q^{2}\right)=i \int d^{4} x e^{i q x}<0\left|T\left(\partial^{\mu} A_{\mu}(x) \partial^{\nu} A_{\nu}^{\dagger}(0)\right)\right| 0>
$$

where $A_{\mu}(x)=: \bar{s}(x) \gamma_{\mu} \gamma_{5} u(x):$, and $\partial^{\mu} A_{\mu}(x)=m_{s}: \bar{s}(x) i \gamma_{5} u(x):$. The QCD expression of this two-point function is known [4], [5], [6] at the four-loop level in perturbative QCD, and up to dimension six in the non-perturbative sector. Also, the old problem of mass singularities has been satisfactorily solved in [4], [0]. As a result of this, quark mass corrections are also known up to quartic order. Notice that the QCD result for the correlator (9) is trivially obtained from that involving the vector current divergences; hence, they both look quite similar. The QCD expression of the Laplace transform of Eq.(9), i.e.

$$
\psi_{5}^{\prime \prime}\left(M^{2}\right)=\hat{L}\left[\psi_{5}^{\prime \prime}\left(Q^{2}\right)\right]=\int_{0}^{\infty} e^{-s / M^{2}} \frac{1}{\pi} \operatorname{Im} \psi_{5}(s) d s
$$

is given by

$\left.\psi_{5}^{\prime \prime}\left(M^{2}\right)\right|_{Q C D}=\left[\bar{m}_{s}\left(M^{2}\right)\right]^{2} M^{4}\left[\psi_{5(0)}^{\prime \prime}\left(M^{2}\right)+\frac{\psi_{5(2)}^{\prime \prime}\left(M^{2}\right)}{M^{2}}+\frac{\psi_{5(4)}^{\prime \prime}\left(M^{2}\right)}{M^{4}}+\frac{\psi_{5(6)}^{\prime \prime}\left(M^{2}\right)}{M^{6}}+\cdots\right]$,

where

$$
\begin{gathered}
\psi_{5(0)}^{\prime \prime}\left(M^{2}\right) \equiv \hat{L}\left[\psi_{5(0)}^{\prime \prime}\left(Q^{2}\right)\right]=\frac{3}{8 \pi^{2}}\left\{1+\frac{\bar{\alpha}_{s}\left(M^{2}\right)}{\pi}\left(\frac{11}{3}+2 \gamma_{E}\right)+\left(\frac{\bar{\alpha}_{s}\left(M^{2}\right)}{\pi}\right)^{2}\left(\frac{5071}{144}\right.\right. \\
\left.-\frac{35}{2} \zeta(3)+\frac{17}{4} \gamma_{E}^{2}+\frac{139}{6} \gamma_{E}-\frac{17}{24} \pi^{2}\right)+\left(\frac{\bar{\alpha}_{s}\left(M^{2}\right)}{\pi}\right)^{3}\left(-\frac{4781}{9}+\frac{1}{6} a_{1}\right. \\
\left.\left.-\frac{475}{4} \zeta(3) \gamma_{E}+\frac{823}{6} \zeta(3)+\frac{221}{24} \gamma_{E}^{3}+\frac{695}{8} \gamma_{E}^{2}-\frac{221}{48} \gamma_{E} \pi^{2}+\frac{2720}{9} \gamma_{E}-\frac{695}{48} \pi^{2}\right)\right\}
\end{gathered}
$$




$$
\begin{gathered}
\psi_{5(2)}^{\prime \prime}\left(M^{2}\right) \equiv \hat{L}\left[\psi_{5(2)}^{\prime \prime}\left(Q^{2}\right)\right]=-\frac{3}{4 \pi^{2}}\left[\bar{m}_{s}\left(M^{2}\right)\right]^{2}\left[1+\frac{\bar{\alpha}_{s}\left(M^{2}\right)}{\pi}\left(\frac{16}{3}+4 \gamma_{E}\right)\right] \\
\psi_{5(4)}^{\prime \prime}\left(M^{2}\right) \equiv \hat{L}\left[\psi_{5(4)}^{\prime \prime}\left(Q^{2}\right)\right]=\frac{1}{8}<\frac{\alpha_{s}}{\pi} G^{2}>+\frac{1}{2}<m_{s} \bar{s} s>\left[1+\frac{\bar{\alpha}_{s}}{\pi}\left(\frac{11}{3}+2 \gamma_{E}\right)\right] \\
-<m_{s} \bar{u} u>\left[1+\frac{\bar{\alpha}_{s}}{\pi}\left(\frac{14}{3}+2 \gamma_{E}\right)\right] \\
+\frac{3}{28 \pi^{2}} m_{s}^{4}\left[-\frac{233}{36}-\frac{15}{2} \gamma_{E}+2 \frac{\bar{\alpha}_{s}}{\pi}\left(\frac{37}{9}+2 \gamma_{E}\right)\left(\frac{\pi}{\bar{\alpha}_{s}}-\frac{53}{24}\right)\right],
\end{gathered}
$$

and where $\gamma_{E}$ is Euler's constant, $\zeta(n)$ is Riemann's zeta function, $a_{1}=2795.0778$, all numerical coefficients refer to three flavours and three colours, and we have neglected the up-quark mass everywhere. Given the uncertainties of the method, plus the size of systematic errors, it is not justified to keep $m_{u}$ different from zero. The four-loop expressions for the strong running coupling and quark mass are given by

$$
\begin{gathered}
\frac{\bar{\alpha}_{s}\left(M^{2}\right)}{\pi}=\frac{4}{9} \frac{1}{L}-\frac{256}{729} \frac{L L}{L^{2}}+\left[6794-16384\left(L L-L L^{2}\right)\right] \frac{1}{59049} \frac{1}{L^{3}} \\
\bar{m}_{s}\left(M^{2}\right)=\frac{\hat{m}_{s}}{\left(\frac{1}{2} L\right)^{4 / 9}}\left\{1+(290-256 L L) \frac{1}{729} \frac{1}{L}+\left[\frac{550435}{1062882}-\frac{80}{729} \zeta(3)\right.\right. \\
\left.-\left(388736 L L-106496 L L^{2}\right) \frac{1}{531441}\right] \frac{1}{L^{2}}+\left[-\frac{126940037}{1162261467}-\frac{256}{177147} \beta_{4}\right. \\
+\frac{128}{19683} \gamma_{4}+\frac{7520}{531441} \zeta(3)+\left(-\frac{611418176}{387420489}+\frac{112640}{531441} \zeta(3)\right) L L \\
\left.\left.+\frac{335011840}{387420489} L L^{2}-\frac{149946368}{1162261467} L L^{3}\right] \frac{1}{L^{3}}\right\}
\end{gathered}
$$

where $L=\log \left(M^{2} / \Lambda_{Q C D}^{2}\right), L L=\log L$, and [14] 


$$
\beta_{4}=-\frac{281198}{4608}-\frac{890}{32} \zeta(3)
$$

with $\gamma_{4}=88.5258$ (see [15]). In line with the discussion after Eq. (7), and following [6], we shall not expand the above QCD expressions in inverse powers of $L$, but rather substitute the numerical values of $\alpha_{s}\left(M^{2}\right)$ and $\bar{m}_{s}\left(M^{2}\right)$ as determined from Eqs.(15)-(16) for a given value of $\Lambda_{Q C D}$. The dimension-six non-perturbative term has been omitted as it is of no numerical importance.

The hadronic spectral function associated with the correlator (9) is very different from that of the vector divergences. There is, at present, preliminary information from tau-decays [13] in a kinematical range restricted by the tau-mass. We reconstruct the spectral function, including in addition to the kaon-pole its radial excitations $\mathrm{K}(1460)$ and $\mathrm{K}(1830)$, normalized at threshold according to conventional chiral symmetry. In addition, we incorporate the resonant sub-channel $K^{*}(892)-\pi$, which is of numerical importance given the narrow width of the $K^{*}(892)$ (the sub-channel $\rho(770)-K$ is numerically negligible). This chiral symmetry normalization is of the form

$$
\left.\frac{1}{\pi} \operatorname{Im} \psi_{5}(s)\right|_{K \pi \pi}=\frac{M_{K}^{2}}{2 f_{\pi}^{2}} \frac{3}{2^{8} \pi^{4}} \frac{I(s)}{s\left(M_{K}^{2}-s\right)} \theta\left(s-M_{K}^{2}\right)
$$

where

$$
\begin{aligned}
I(s)= & \int_{M_{K}^{2}}^{s} \frac{d u}{u}\left(u-M_{K}^{2}\right)(s-u)\left\{\left(M_{K}^{2}-s\right)\left[u-\frac{\left(s+M_{K}^{2}\right)}{2}\right]\right. \\
& \left.-\frac{1}{8 u}\left(u^{2}-M_{K}^{4}\right)(s-u)+\frac{3}{4}\left(u-M_{K}^{2}\right)^{2}\left|F_{K^{*}}(u)\right|^{2}\right\},
\end{aligned}
$$

and

$$
\left|F_{K^{*}}(u)\right|^{2}=\frac{\left[M_{K^{*}}^{2}-M_{K}^{2}\right]^{2}+M_{K^{*}}^{2} \Gamma_{K^{*}}^{2}}{\left(M_{K^{*}}^{2}-u\right)^{2}+M_{K^{*}}^{2} \Gamma_{K^{*}}^{2}} .
$$

The pion mass has been neglected above, in line with the approximation $m_{u}=0$ made in the QCD sector, and in our normalization $f_{\pi} \simeq 93 \mathrm{MeV}$. The complete hadronic spectral function is then 


$$
\begin{aligned}
\left.\frac{1}{\pi} \operatorname{Im} \psi_{5}(s)\right|_{H A D}=2 f_{K}^{2} M_{K}^{4} & \delta\left(s-M_{K}^{2}\right)+\left.\frac{1}{\pi} \operatorname{Im} \psi_{5}(s)\right|_{K \pi \pi} \frac{\left[B W_{1}(s)+\lambda B W_{2}(s)\right]}{(1+\lambda)} \\
+ & \left.\frac{1}{\pi} \operatorname{Im} \psi_{5}(s)\right|_{Q C D} \theta\left(s-s_{0}\right)
\end{aligned}
$$

where $f_{K} \simeq 1.2 f_{\pi},\left.\operatorname{Im} \psi_{5}(s)\right|_{Q C D}$ is the perturbative QCD spectral function modelling the continuum which starts at some threshold $s_{0}, B W_{1,2}(s)$ are Breit-Wigner forms for the two kaon radial excitations, normalized to unity at threshold, and $\lambda$ controls the relative importance of the second radial excitation. The choice $\lambda \simeq 1$ results in a reasonable (smaller) weight of the $\mathrm{K}(1830)$ relative to the $\mathrm{K}(1460)$.

We have solved the Laplace transform QCD sum rules using the values: $<\alpha_{s} G^{2}>\simeq$ $0.024 \mathrm{GeV}^{4},<\bar{s} s>\simeq<\bar{u} u>=-0.01 \mathrm{GeV}^{3}$, and allowing $\Lambda_{Q C D}$ and $s_{0}$ to vary in the range: $\Lambda_{Q C D}=280-380 \mathrm{MeV}$, and $s_{0}=4-8 \mathrm{GeV}^{2}$. The results for $m_{s}\left(1 \mathrm{GeV}^{2}\right)$ are very stable against variations in the Laplace variable $M^{2}$ over the wide range: $M=1-4 \mathrm{GeV}^{2}$. In Figures 1 and 2 we show some typical results for $m_{s}\left(1 \mathrm{GeV}^{2}\right)$ as a function of $M^{2}$ for, respectively, $\Lambda_{Q C D}=280 \mathrm{MeV}, s_{0}=4-6 \mathrm{GeV}^{2}$, and $\Lambda_{Q C D}=380 \mathrm{MeV}, s_{0}=6-8 \mathrm{GeV}^{2}$. Combining all results gives

$$
\bar{m}_{s}\left(1 \mathrm{GeV}^{2}\right)=155 \pm 25 \mathrm{MeV} .
$$

This result is consistent with the other determinations in the scalar channel, Eqs.(6)-(8). The error given above originates exclusively from changes in the relevant parameters, and does not reflect possible systematic uncertainties from the hadronic sector. 


\section{Figure Captions}

Figure 1. The running strange quark mass $m_{s}\left(M^{2}=1 \mathrm{GeV}^{2}\right)$ as a function of the Laplace variable $M^{2}$, for $\Lambda_{Q C D}=280 \mathrm{MeV}$. Upper and lower curves determine the range obtained by varying $s_{0}$ in the interval: $s_{0}=4.0-6.0 \mathrm{GeV}^{2}$.

Figure 2. The running strange quark mass $m_{s}\left(M^{2}=1 \mathrm{GeV}^{2}\right)$ as a function of the Laplace variable $M^{2}$, for $\Lambda_{Q C D}=380 \mathrm{MeV}$. Upper and lower curves determine the range obtained by varying $s_{0}$ in the interval: $s_{0}=6.0-8.0 \mathrm{GeV}^{2}$. 


\section{References}

[1] S.Narison, N.Paver, E.de Rafael, and D.Treleani, Nucl. Phys. B 212 (1983) 365; C.A.Dominguez and M.Loewe, Phys.Rev. D 31 (1985) 2930.

[2] C.A.Dominguez and E.de Rafael, Ann. Phys. (N.Y.) 174 (1987) 372.

[3] C.A.Dominguez, C.van Gend, and N.Paver, Phys. Lett. B 253 (1991) 241.

[4] K.G.Chetyrkin, C.A.Dominguez, D.Pirjol, and K.Schilcher, Phys.Rev. D 51 (1995) 5090.

[5] M.Jamin and M.Münz, Z. Phys. C 66 (1995) 633.

[6] K.G.Chetyrkin, D.Pirjol, and K.Schilcher, Phys. Lett. B 404 (1997) 337.

[7] P.Colangelo, F.De Fazio, G.Nardulli, and N.Paver, Phys. Lett. B 408 (1997) 340.

[8] J.Bijnens, J.Prades, and E.de Rafael, Phys. Lett. B 348 (1995) 226.

[9] H.Leutwyler, Phys. Lett. B378 (1996) 313.

[10] M.Knecht, H.Sazdjian, and J.Stern, Phys. Lett. B 313 (1993) 229.

[11] D. Aston et al., Nucl. Phys. B 296 (1988) 493.

[12] R.M.Barnett et al., Phys. Rev. D 54 (1996) 1.

[13] M.Davier, Orsay Report No. LAL-96-94 (1996).

[14] T. van Ritbergen, J.A.M. Vermaseren, and S.A. Larin, Phys. Lett. B 400 (1997) 379.

[15] A.L. Kataev and V.V. Starschenko, Mod. Phys. Lett. A10 (1995) 235. 
This figure "fig1-1.png" is available in "png" format from: http://arxiv.org/ps/hep-ph/9712369v1 
This figure "fig1-2.png" is available in "png" format from: http://arxiv.org/ps/hep-ph/9712369v1 